\title{
Vibration analysis based on surface acoustic wave sensors
}

Alfred P. Gnadinger ${ }^{1}$

'Albido Corporation, Colorado Springs, CO 80906, USA

\begin{abstract}
It is important to know, whether a civil engineering structure is safe or unsafe. One way to determine this is to measure vibrations at critical locations and feeding this data into an appropriate algorithm. Albido Corporation has developed wireless strain sensors based on surface acoustic wave (SAW) principles that are mainly employed on rotating structures and in harsh environments. Albido's sensors could also be used to measure vibrations in civil engineering structures. They are small $(\sim 1 \times 3$ $\mathrm{mm})$, passive and inexpensive $(<1 \$$ in volume). They are powered by the electromagnetic field emanating from the antenna of a Reader System, similar to an RFID. The Reader System is essentially a computer with special software and has signal processing capability. One Reader System can service a multitude of sensors. The Reader antenna has to be within the reading range of the sensor. If large distances are required, a small electronic component acting as a Reader System can be placed within the reading range of the sensor that receives the sensor signal, generates a radio signal and encodes the sensor information on the radio signal. Then, the final data processing center can be placed anywhere.
\end{abstract}

\section{Introduction}

There is a need to determine whether a civil engineering structure such as a bridge, tunnel or dam is safe or unsafe. One way to determine this is to measure vibrations at critical points and feed this data into an appropriate algorithm. Critical points can be anywhere and are best determined by a civil engineer or a person responsible for the safety of a civil engineering structure. The vibration analysis should be ubiquitous, that means, it should be independent of sensor locations. The vibration sensors should ideally be wireless since it is cumbersome or often impossible to route wires from the sensors to the data processing unit. Some wireless telemetry systems have been proposed in the past $[1,2]$, but all of them have some drawbacks (e.g. bulky, lack of flexibility, need for power supply, short operating distance, etc.) making them inappropriate for most applications. The data processing unit quite often is hundreds of meters away from the vibration sensors and a multitude of sensors (tens if not hundreds) should be serviced by a single data processing center. We assumed a bridge as the object of investigation. However, the ideal vibration analysis technology should be independent of the particular structure. It should be applicable also to civil engineering structures such as tunnels, highways, airport runways, and others. SAW devices can also be embedded in the structure since civil engineering structures are typically non-metallic (non-conductive). Again, wired approaches are cumbersome or impossible and the distance between the sensors and the data processing center can be very large, quite often hundreds of meters or even kilometers. We are not trying to invent a new way to ascertain the safety of a civil engineering structure but simply offer a novel sensor system to collect and transmit vibration data to a data processing center which we call a Reader.

The most important parameters of vibrations are acceleration, amplitude and frequency. Albido's sensors essentially measure strain. We assume that there is a correlation between acceleration, amplitude and strain. The vibration data we are transmitting are strain and frequency. It should be noted here, that the proposed method is strictly a different method to measure and transmit vibration data. Specifically, we propose to use surface acoustic wave (SAW) sensors to characterize vibrations. We do not intend to alter the way a civil engineering structure is characterized for safety.

Albido Corporation, a US Corporation headquartered in Colorado Springs CO, has developed a suite of wireless strain sensors based on surface acoustic wave (SAW) principles. Albido's sensors are currently employed in harsh environments and under circumstances where connecting wires is not feasible. Albido works mostly for the US government and currently has one Department of Defense contract (US Navy) for employing Albido's sensors as torque sensors on the rotating shafts of large pumps and other Navy equipment and one with NASA to measure and monitor strain in the hulls of rocket vehicles and other NASA space and aeronautical equipment.

Albido's strain sensors are small (e.g. $\sim 1 \times 3 \mathrm{~mm}$ ), wireless and passive. That means they do not require a power source such as a battery, but are powered by the electromagnetic field emanating from the antenna of the data processing system, similar to an RFID. The distance between the antenna of the sensor and the antenna of the Data Processing Center (Reader) is the "reading range" of about $10 \mathrm{~cm}$ to about 1 meter, depending on frequency and other parameters. This is probably too short for the application of characterizing vibrations on civil engineering structures. However, if the distance between the sensor most removed to the Reader is less than about 10 meters, the operating frequency can be increased so that the reading range is about 10 meters and no intermediary electronic component is necessary. In most cases, however, the reading range will be more than 10 meters and an additional electronic component acting as a Reader is needed. This component will consist of a reader antenna, a power source (e.g. a battery), limited data processing capability, and, most importantly, a means to generate a radio wave (for example, in the MHz/FM band range). The strain data collected from the sensors is now encoded on this radio wave and transmitted to a central data processing facility that can be kilometers away. We expect the electronic component to be essentially a printed circuit board, about $5 \mathrm{~cm} \mathrm{x} 15 \mathrm{~cm}$ large, with an antenna and a battery connected to it. A possible implementation is shown in Fig. 1.

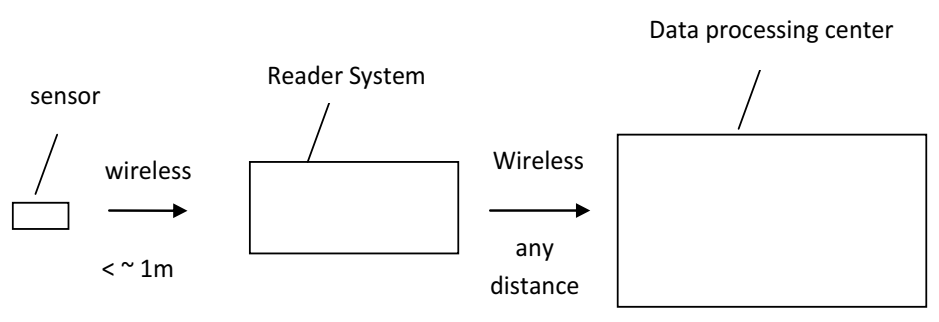

Fig. 1 Two stage transmission scheme if more than about 1 meter reading range is required.

Albido's strain sensors are small (about 1 x $3 \mathrm{~mm}$ ) and inexpensive (less than $1 \$$ in volume). One reader system (data processing center) can service a multitude of sensors. In order to accomplish this, we use anti-collision technology such as various modulation schemes (e.g. TDMA (Time Division Multiplexing Access), FDMA (Frequency DMA), CDMA (Code DMA), OFC (Orthogonal Frequency Coding), or SDMA (Spatial DMA)). The Auto ID Center research consortium formed at MIT is developing a standard for networks of passive RFID sensors - as opposed to active devices used in Bluetooth or ZigBee networks. If appropriate, we will adhere to that standard.

We estimate that about $10-20$ sensors can be serviced by a single Reader system. If more sensors are required, more than one Reader system is needed. In the current application to characterize vibrations in civil engineering structures, we anticipate one or more reader systems and one central data processing system a certain distance away. 
A SAW sensor is not only sensitive to the physical parameter to be measured such as strain but also to temperature. Albido has developed and patented (US patent $8,258,674$, issued on 4 Sep 2012) a method to distinguish between strain and temperature [3]. In fact, the scheme developed by Albido allows the measurement of temperature. The method is quite simple. Since the sensors are inexpensive there is no problem to have two sensors instead of one at a given critical point. In order to separate the temperature and strain response, the sensors are built slightly differently (e.g. different electrode width or thickness, different substrate material, etc.). The sensors are very close to each other, so we can assume that the temperature is the same. The sensor response to strain (or vibration in our case), however, is slightly different. Some signal processing is done in the Reader system and the strain response is calculated independent of temperature.

\section{Surface acoustic wave (SAW) devices}

In recent years, important progress has been made in wireless SAW sensors yielding a variety of innovative applications. These developments are mainly based upon the implementation of SAW radiofrequency identification (RFID) labels and their combination with traditional SAW sensor techniques for physical or chemical parameters $[4,5]$. The operating principle of these systems is shown schematically in figure 4. A radio frequency (RF) electromagnetic request signal is transmitted by the radar-like transceiver (Trx) and is picked up by the antenna of the passive SAW transponder where an interdigital transducer (IDT), connected to the antenna, converts the received signal into a surface acoustic wave (SAW). It is important to realize that the frequencies of the electromagnatic wave and the surface acoustic wave are orders of magnitude apart. The frequency of the electromagnetic wave is typically in the MHZ range whereas the acoustic wave frequency is at most in the $\mathrm{kHz}$ range. The acoustic wave propagates along the surface and is partially reflected at each reflector. The reflector can be a metalization or simply a discontinuity (e.g. a groove) in the substrate. Typically about 100 reflectors are used to achieve sufficient efficieny. The acoustic wave packets returning to the IDT are re-converted into electrical signals and re-transmitted to the reader (Trx) unit by the transponder antenna shown by example as a dipole antenna in Figure 2. This response contains information about the number and location of reflectors as well as the propagation and the reflection properties of the SAW. Its evaluation in the signal processing unit of the reader will allow for the extraction of the desired information, for example the sensor effect for a certain parameter and/or a specific identification number.

The range of a surface wave transponder system can be determined using the radar equation. The influence of coherent averaging is taken into account as "integration time":

$$
d=\sqrt[4]{\frac{P_{T} \cdot G_{T}^{2} \cdot G_{S}^{2} \cdot t_{i} \cdot \lambda^{4}}{k \cdot T_{0} \cdot B \cdot F \cdot(S / N) \cdot I L}}
$$

where $d$ is the reading range, $P_{T}$ is the transmitter power, $G_{T}$ is the transmitter antenna gain, $G_{S}$ is the sensor antenna gain, $\lambda$ is the RF signal wavelength, $B$ is the reader bandwidth, $F$ is the reader noise, $S / N$ is the signal to noise ratio, $I L$ is the transponder insertion loss, $t_{i}$ is the integration time, $k$ is Boltzmann's constant, and $T_{o}$ is the temperature. It can be shown that a reading range of about 1 meter can be achieved at $433 \mathrm{MHz}$ or between 0.2 to 2 meters at $2.45 \mathrm{GHz}$ if an omnidirectional transmitter antenna (unity gain) is used and depending on the integration time $[4,6]$. However, the reading range can be increased for low data rates and by using a directional antenna and appropriate encoding techniques.

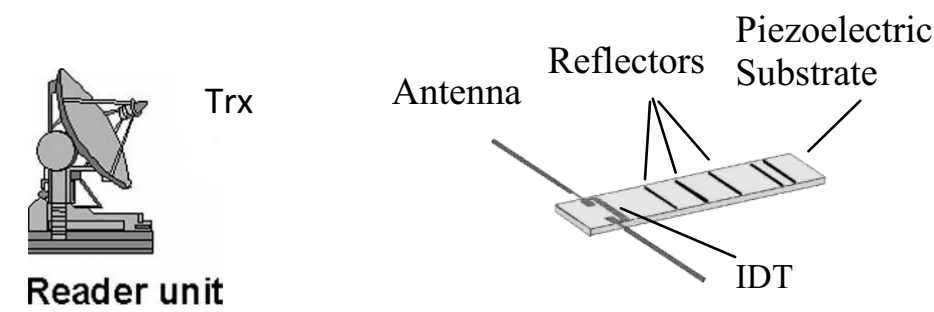

Figure 2. Schematic of a SAW-based radio-link measurement system

If a voltage is applied to the electrodes of a piezoelectric crystal such as quartz $\left(\mathrm{SiO}_{2}\right)$, lithium niobate $\left(\mathrm{LiNbO}_{3}\right)$, or lithium tantalite $\left(\mathrm{LiTaO}_{3}\right)$, mechanical distortions arise in the crystal lattice as a result of the piezoelectric effect. This effect is used to generate surface acoustic waves (SAW) on the crystal. To achieve this, electrode structures made of a conductor, approximately $0.1 \mu \mathrm{m}$ thick (aluminum, gold, platinum, et al.) are applied to the polished surface of a piezoelectric single crystal in the form of an electro acoustic converter. When an alternating voltage is applied to the electro acoustic converter, surface acoustic waves propagate on the surface of the crystal. Interdigital electrode structures in the form of interleaved fingers make effective electro acoustic transducers. Figure 3 shows a typical acoustic wave device consisting of two sets of interdigital transducers (IDT), one transducer converts electric field energy into mechanical wave energy; the other converts the mechanical energy back into an electric field. Instead of two IDT structures, SAW sensors with only one such structure but multiple reflectors as shown in Figure 4 are feasible. These resonator-types SAW devices are used by Albido and proposed in the current proposal.

The distance between two fingers of the same polarity is termed the electrical period $\mathrm{q}$ of the IDT. The maximum electro-acoustic interaction is obtained at the frequency $\mathrm{f}_{0}$, the mid-frequency of the transducer. At this frequency the wavelength $\lambda_{0}$ of the surface acoustic wave precisely corresponds to the electrical period $q$ of the IDT, so that all wave trains are superimposed in-phase and transmission is maximized $\mathrm{q}=\lambda_{0}=\mathrm{v} / \mathrm{f}_{0}$. The relationship between the electrical and mechanical power density of a surface wave is described by the material-dependent piezoelectric coupling coefficient $\mathrm{k}^{2}$. Around $1 / \mathrm{k}^{2}$ overlaps of the transducer are required to convert the entire electrical power applied to the IDT into the acoustic power of a surface wave. For quartz $\mathrm{k}^{2}=0.16 \%$ or $0.0016 .1 / \mathrm{k}^{2}=625$. Of the order of 600 overlaps are, therefore, required to convert the entire electrical power applied to the IDT. We expect to use about 500 fingers in our resonant type SAW sensors.

The velocity $v$ of a surface wave on the substrate, and thus the propagation time $\tau$ and the mid-frequency $f_{0}$ of a surface wave component, can be influenced by a range of physical variables. In addition to temperature, mechanical forces such as static elongation, compression, shear, bending and acceleration have a particular influence upon the surface wave velocity. This facilitates the remote interrogation of mechanical forces by surface wave sensors. In general, the sensitivity $S$ of the quantity $x$ to a variation of the influence quantity $y$ can be defined as:

$$
\mathrm{S}_{\mathrm{y}}^{\mathrm{x}}=\frac{1}{\mathrm{x}} \frac{\partial \mathrm{x}}{\partial \mathrm{y}}
$$

To first order, the influence of the quantity $y$ (which can be temperature or strain,) on the mid-frequency $f_{0}$ and propagation time $\tau$ can be calculated as follows: 


$$
\begin{aligned}
& v(y)=v\left(y_{0}\right) \cdot\left[1-S_{y}^{v} \cdot\left(y-y_{0}\right)\right] \\
& f_{0}(y)=f_{0}\left(y_{0}\right) \cdot\left[1-S_{y}^{f} \cdot\left(y-y_{0}\right)\right] \\
& \tau(y)=\tau\left(y_{0}\right) \cdot\left[1+S_{y}^{\tau} \cdot\left(y-y_{0}\right)\right]
\end{aligned}
$$

If only the differential propagation times or the differential phase between the individual reflected pulses are evaluated, the sensor signal is independent of the distance between the reader and the transponder. The differential propagation time $\tau_{2-1}$, and the differential phase $\varphi_{2-1}$ between the two received pulses is obtained from the distance $L_{2-1}$ between the two reflectors, the velocity $v$ of the surface wave, and the frequency $f$ of the interrogation pulse.

$$
\tau_{2-1}=\frac{2 \cdot L_{2-1}}{v} ; \quad \varphi_{2-1}=2 \pi f \cdot \tau_{2-1}=\frac{4 \pi f \cdot L_{2-1}}{v}
$$

The measurable change $\Delta \tau_{2-1}$ or $\Delta \varphi_{2-1}$ when a physical quantity $y$ is changed by the amount $\Delta y$ is thus:

$$
\Delta \tau_{2-1}=\tau_{2-1} \cdot S_{y}^{\tau} \cdot \Delta y ; \quad \Delta \varphi_{2-1}=2 \pi f \cdot \tau_{2-1} \cdot S_{y}^{\tau} \cdot \Delta y
$$

The influence of the physical quantity $y$ on the surface wave transponder can thus be determined only by the evaluation of the phase difference between the different pulses of the response signal.

In a reflective delay line the available path is used twice. However, if the IDT is positioned between two fully reflective structures, then the acoustic path can be used many more times due to multiple reflections. Such an arrangement is called a surface wave one-port resonator and is preferably used by Albido. The distance between the two resonators must be an integer multiple of the half wavelength $\lambda_{0}$ at the resonant frequency $f_{1}$. The displacement of the mid-frequency $\Delta f_{1}$ and the displacement of the associated phase $\Delta \varphi_{1}$ of a resonator due to a change of the physical quantity $y$ with loaded $Q$ factor are:

$\Delta f_{1}=-f_{1}\left(y_{0}\right) \cdot S_{y}^{f} \cdot \Delta y ; \quad \Delta \varphi=2 Q \cdot \frac{\Delta f_{1}}{f_{1}}$

where $f_{1}$ is the unaffected resonant frequency of the resonator.

From the equations above it is obvious that the influence quantity $y$ can be estimated by measuring time delay $(\tau)$, phase $(\varphi)$, or frequency $(f)$ variation. Temperature, strain (or pressure), and other parameters can be measured with very good accuracy $\left(0.1{ }^{0} \mathrm{C}, 1\right.$ $\mu$ strain, etc.) at very high rate (up to $10^{5}$ measurements per second, depending on the resonant frequency). The measurement is essentially static, but since it can be performed very quickly, it can also catch the variation (oscillatory or not, if any) of the measured parameters.

SAW sensors have some special features which distinguish them from other kinds of sensors. They are very small $\left(<4 \mathrm{~mm}^{3}\right.$ volume), very light $(<1$ gram), low cost (only one or at most two patterned photolithography step needed), and they can operate in very harsh environments $\left(-50^{\circ} \mathrm{C}\right.$ to $+500^{\circ} \mathrm{C}$, depending on the piezoelectric substrate). Thin layer piezoelectric materials (single or polycrystalline) are commonly used for radiofrequency resonators and ultrasonic actuators/transducers where they vibrate at high frequency $(>1 \mathrm{MHz})$ for long periods of time. So, as long as the elasticity limits are not exceeded, it has been proved that the piezoelectric devices (SAW included) can withstand a very large number of cycles $\left(>10^{12}\right)$ without pronounced fatigue effects. It has also been shown that SAW devices are radiation hard ( $>10 \mathrm{M}$ Rad Gamma) and behave very well when in close proximity to metallic objects. We believe that they are well suited for the current application to characterize vibrations in civil engineering structures.

As mentioned before, there are essentially two types of SAW sensors: The first one is a four terminal device where there are two distinct IDT's (Interdigitated Structures), one as input where the input electromagnetic wave is converted into a SAW and a second IDT, a certain distance away from the first IDT, where the SAW is reconverted into an electromagnetic wave. The second one is a two terminal device with only one IDT (both for input and output) but with a multitude of reflectors on both sides of the IDT. This device is operated in a resonant mode. The change in resonant frequency is correlated to a change in physical parameters. Fig. 3 and Fig. 4 show these two types of SAW. Albido prefers to use the resonant device of Fig. 4.

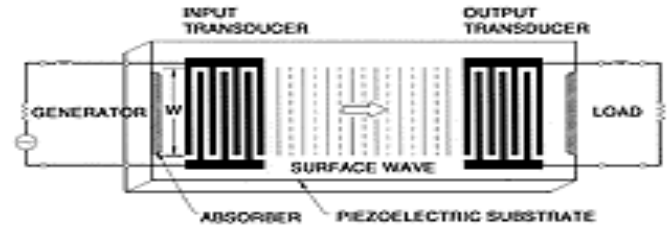

Fig 3. Two port (four terminal) SAW sensor

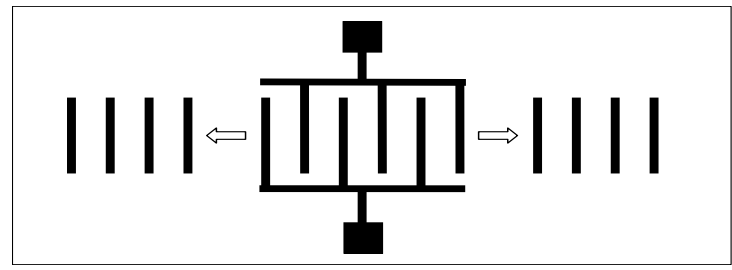

Fig. 4. One port (two terminal) SAW sensor

As mentioned before, the reading range of a typical SAW sensor is typically less than about 1 meter, depending on frequency and other parameters. For the application to measure vibrations in civil engineering structures we expect a much longer reading range. In that case a two way communication scheme is needed. We propose to place a battery powered Reader within the reading range and transmit the data to a remote signal processing unit that can be kilometers away.

\section{Results of Albido's wireless strain sensors}

Fig. 5 shows a typical response of an Albido strain sensor. We plotted the change in resonant frequency (in $\mathrm{MHz}$ ) of an experimental SAW sensor as a function of strain (in $\mu$ strain).

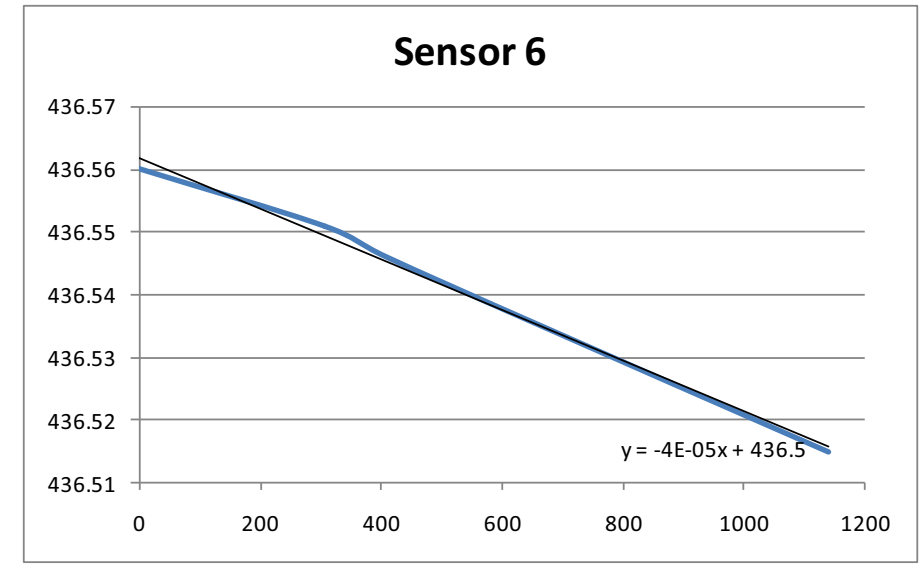

Fig. 5 Typical SAW strain sensor response of an Albido sensor

It can be seen in Fig. 5 that the response is fairly linear given by:

$$
y=436.56-4 E-05 x
$$

Fig. 6 is a picture of an experimental Albido strain sensor. We operate in the mid $\mathrm{MHz}$ range (e.g. $415 \mathrm{MHz}$, a frequency allowed in the USA). The sensor antenna size is a strong function of frequency. In general, the higher the frequency the smaller the antenna. The sensor itself is on top of Fig. 6 and connected to fairly large structures acting as bond pads with two thin wires. The large 
bond pads are strictly to ease connections in a laboratory environment and are not present in commercial devices. These sensors can easily be adapted to measure vibrations, the topic of this paper. We expect data with low frequencies, certainly less than about $20 \mathrm{kHz}$, the current upper limit of Albido's SAW sensors. SAW sensors are very sensitive and fast. Measuring vibrations is well within the capability of SAW sensors.

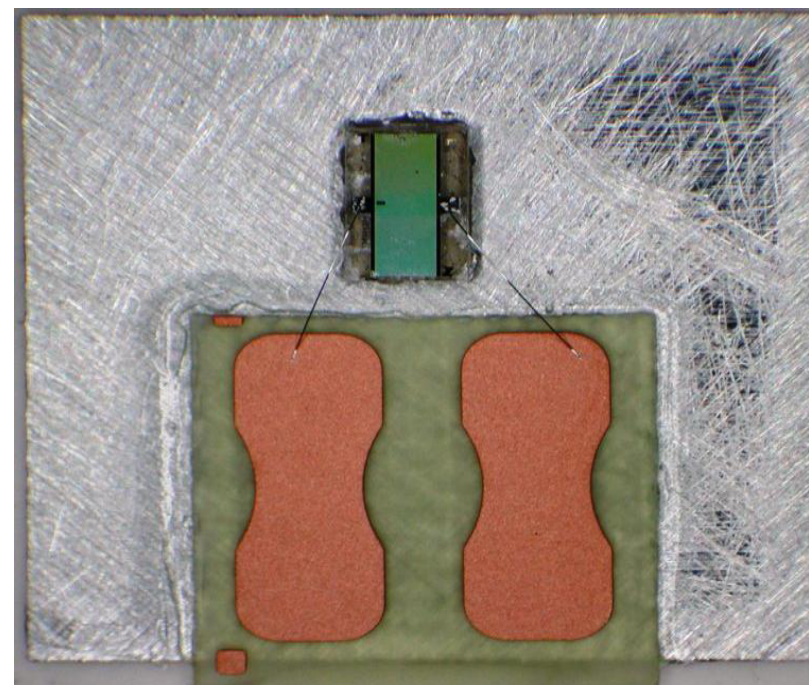

Fig. 6. Experimental Albido strain sensor

\section{Conclusions and Summary}

We propose to use Albido's strain sensors to characterize vibrations in civil engineering structures.

Albido's sensor are wireless, passive, small ( 1 $\mathrm{mm} \times 3 \mathrm{~mm})$, light weight $(<1 \mathrm{~g})$ and inexpensive $(<1 \$$ in volume). So far they have been used to measure torque and rate of change of torque in rotating machinery (Navy) and vibrations and strain in enclosures of space and other areonautical structures (NASA).

We believe that Albido's sensors can also be used to measure and monitor vibrations in civil engineering structures such as bridges, dams, tunnels, highways, airport runways and others. If more than about 1 meter reading range is required (very likely) a two way communication scheme with a small electronic component as part of the second stage can be used, or, if the reading range is less than about 10 meters, a high frequency can be used to maximize reading range.

All SAW sensors are sensitive to temperature changes in addition to the physical parameters to be measured. Albido has invented and patented a scheme to separate the effects of temperature and other physical parameters such as strain.

\section{References}

1. S.W.Arms, C.P.Townsend, J.H.Galbreath, A.T.Newhard, Wireless Strain Sensing Networks, 2nd European Workshop on Structural Health Monitoring, (July 2004).

2. DeAnna, G. Russell, Wireless Telemetry for Gas Turbine Application, U.S.Army Research Laboratory, (March 2000).

3. Viorel Olariu, Surface acoustic wave sensor and system, US Patent 8,258,674 (2012).

4. K.Finkenzeller, RFID Handbook, J.Wiley \& Sons (2003).

5. P.A.Nysen, H.Skeie, D.Armstrong, U.S.Patent $4,725,841$ (1988).

6. R.W.Brocato, Passive wireless sensor tags, Sandia National Laboratory Report (2006). 